\begin{tabular}{|c|c|c|}
\hline 1 & CWAT & 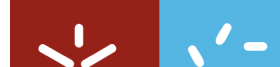 \\
\hline - Reprositón & Centro de Matemática da Universidade do Minho & -1 \\
\hline \multirow[t]{2}{*}{ Universidade do Minho } & Campus de Gualtar 4710-057 Braga Portugal & \multirow{2}{*}{$\begin{array}{l}\text { Universidade do Minho } \\
\text { Escola de Ciências } \\
\text { Centro de Matemática }\end{array}$} \\
\hline & www.cmat.uminho.pt & \\
\hline
\end{tabular}

\title{
Quaternionic polynomials with multiple zeros: a numerical point of view
}

\author{
M. Irene Falcão ${ }^{a, c} \quad$ Fernando Miranda $a^{a, c} \quad$ Ricardo Severino $^{c} \quad$ M. Joana Soares ${ }^{b, c}$ \\ a CMAT - Centre of Mathematics, University of Minho, Portugal \\ ${ }^{b}$ NIPE - Economic Policies Research Unit, University of Minho, Portugal \\ ${ }^{c}$ Department of Mathematics and Applications, University of Minho, Portugal
}

\section{Information}

Keywords:

Quaternionic polynomials, Zeros, Multiplicity.

Original publication:

AIP Conference Proceedings

1798(1), 020099 (2017)

DOI: $10.1063 / 1.4972691$

American Institute of Physics

\begin{abstract}
In the ring of quaternionic polynomials there is no easy solution to the problem of finding a suitable definition of multiplicity of a zero. In this paper we discuss different notions of multiple zeros available in the literature and add a computational point of view to this problem, by taking into account the behavior of the well known Newton's method in the presence of such roots.
\end{abstract}

\section{Introduction}

There are a lot of root-finding algorithms especially designed for real or complex polynomials. However, many methods face difficulties in dealing with multiple zeros or clusters of zeros, since the convergence slows down to order one in such cases (see e.g. [1]).

Because of the non-commutativity of quaternion multiplication, one can consider different classes of polynomials in one quaternion variable, depending on whether a variable is assumed to commute with the polynomial coefficients or not. In the ring of quaternionic polynomials, where the multiplication is defined as in the commutative case, the problem of finding a natural definition of multiplicity for zeros of quaternionic polynomials is a rather complicated task. Thus we can foresee further difficulties in computing multiple quaternionic roots and identifying the multiplicities. A first attempt to handle multiple-root issues was carried out in [2], where special polynomials over $\mathbb{H}$ and quaternionic versions of Newton's method were considered. The objective of this paper is to use the behavior of the classical Newton method as a tool for discussing multiplicity concepts that we have found in the literature $[3,4,5,6,7,8,9]$.

The paper is organized as follows: after recalling in Section 2 the classical multiple-root issues, we introduce in Section 3 the fundamentals of the ring of quaternionic polynomials. We have collected different notions of multiplicity we came across in the literature and present them in Section 4. Finally, in Section 5 we illustrate the computational point of view of Newton's method, by considering its behavior for several different polynomials. 


\section{Recalling the classical cases}

In the ring of complex polynomials $\mathbb{C}[x]$, the notion of multiple root is well known and understood: an element $r \in \mathbb{C}$ is a root of multiplicity $m \in \mathbb{N}$ of $P \in \mathbb{C}[x]$ if there is a polynomial $Q \in \mathbb{C}[x]$ such that $Q(r) \neq 0$ and

$$
P(x)=(x-r)^{m} Q(x) .
$$

Alternatively, we can also define the multiplicity of $r$ as the least nonnegative integer $m$ such that the $m$-th derivative of $P$ does not vanish at $r$.

One of the most popular root-finding methods is Newton's method which consists on approximating a real or complex zero $x^{*}$ of a polynomial $P \in \mathbb{C}[x]$, by means of the iterative process

$$
\text { C-NM : } \quad z_{k+1}=z_{k}-\frac{P\left(z_{k}\right)}{P^{\prime}\left(z_{k}\right)}, k=0,1, \ldots,
$$

with $z_{0}$ sufficiently close to $x^{*}$ and $P^{\prime}\left(z_{k}\right) \neq 0$. This method is very efficient near a simple root, as it exhibits quadratic convergence in a neighborhood of such zero, but in the presence of a multiple root the rate of convergence of the method is only linear. Therefore, the multiplicity of the roots plays an important role in the convergence of Newton's method.

To make clear this fact, we consider polynomials $P_{i}$ whose roots are $r_{1}=0$ with multiplicity $m_{1}=1$ and $r_{2}=\mathbf{i}$ with multiplicity $m_{2}=i ; i=1, \ldots, 4$ and present, in Table 1 , the basins of attraction of those roots, illustrating in this way the classical behavior of Newton's method.

Basins of attraction are an important tool to visually understand how a root-finding method behaves as a function of the initial guesses and have been used, e.g. in [10] as a criterion for comparison of various methods and in [11] to analyze the influence of the multiplicities of the roots in the corresponding basins of attraction when Newton's method is applied to complex polynomials of the form $\left(x-\alpha_{1}\right)^{m_{1}}\left(x-\alpha_{2}\right)^{m_{2}}$ (see also the references therein). The idea is the following: initial points in a bounded region in the complex plane are selected and are then colored according to which root they converge to (see [12] for other details). In our case, the initial points were selected in the square $[-2,2] \times[-2,2]$, red was used for coloring initial guesses converging to 0 and blue for points converging to i. Furthermore, we use color intensity to reflect the number of iterations necessary to converge to a root, with points requiring more iterations appearing with less intensity. We can observe that the speed of convergence to the multiple root $\mathbf{i}$ gets slower (more iterations are required, i.e. lighter shade of blue in the picture) as the multiplicity gets higher. On the other hand, the number of starting points converging to the multiple root increases with the multiplicity i.e. the basin of attraction of $\mathbf{i}$ "invades" more and more the basin of attraction of the simple root 0 , as $i$ increases.

Table 1: Newton's method applied to polynomials with a multiple root
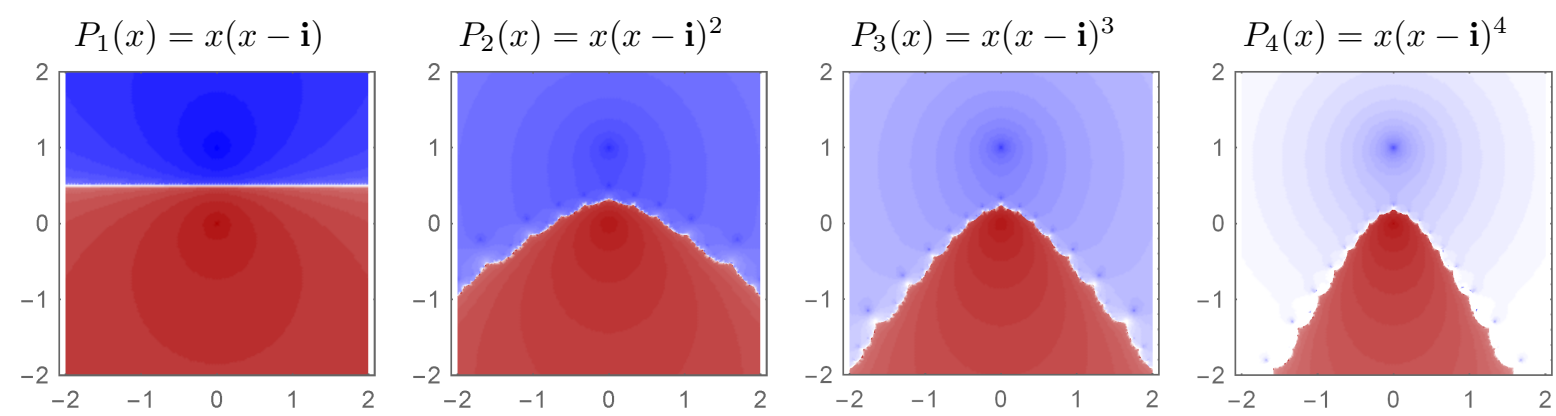

Writing $P(z)=P(x+i y)=u(x, y)+i v(x, y)$, where $u$ and $v$ are real valued functions and identifying the complex number $z=x+i y$ with the vector $z=(x, y) \in \mathbb{R}^{2}$, the equation $P(z)=0$ in the complex variable $z$ can be written as a system of two equations in two real unknowns, namely $\boldsymbol{P}(\boldsymbol{z})=(u(\boldsymbol{z}), v(\boldsymbol{z}))=(0,0)$, which can be solved by applying the classical 2D-Newton method

$$
\text { 2D-NM : } \quad \boldsymbol{z}_{k+1}=\boldsymbol{z}_{k}-\left(J \boldsymbol{P}\left(\boldsymbol{z}_{k}\right)\right)^{-1} \boldsymbol{P}\left(\boldsymbol{z}_{k}\right), k=0,1, \ldots,
$$


provided that the Jacobian matrix $J \boldsymbol{P}\left(\boldsymbol{z}_{k}\right)$ is nonsingular. Moreover, since the function under consideration is a complex polynomial, the complex Newton method coincides with the corresponding 2D-Newton method and $\operatorname{det}(J \boldsymbol{P}(\boldsymbol{z}))=\left|P^{\prime}(z)\right|$ (see [13] for details).

\section{Polynomials in $\mathbb{H}[x]$}

Let $\{1, \mathbf{i}, \mathbf{j}, \mathbf{k}\}$ be an orthonormal basis of the Euclidean vector space $\mathbb{R}^{4}$ with a product given according to the multiplication rules

$$
\mathbf{i}^{2}=\mathbf{j}^{2}=\mathbf{k}^{2}=-1, \quad \mathbf{i j}=-\mathbf{j i}=\mathbf{k} .
$$

This non-commutative product generates the well known algebra of real quaternions $\mathbb{H}$. The real vector space $\mathbb{R}^{4}$ is embedded in $\mathbb{H}$ through the identification of the element $\boldsymbol{x}=\left(x_{0}, x_{1}, x_{2}, x_{3}\right) \in \mathbb{R}^{4}$ with the element $x=x_{0}+x_{1} \mathbf{i}+x_{2} \mathbf{j}+x_{3} \mathbf{k} \in \mathbb{H}$. The conjugate of $x$ is given by $\bar{x}:=x_{0}-x_{1} \mathbf{i}-x_{2} \mathbf{j}-x_{3} \mathbf{k}$, the real part of the quaternion $x$ is $\operatorname{Re} x:=\frac{1}{2}(x+\bar{x})=x_{0}$ and the vector part is $\operatorname{Vec} x:=\frac{1}{2}(x-\bar{x})=x_{1} \mathbf{i}+x_{2} \mathbf{j}+x_{3} \mathbf{k}$. The norm $|x|$ of $x$ is defined by $|x|^{2}=x \bar{x}=\bar{x} x$ and coincides with the Euclidean norm of $\boldsymbol{x}$. It follows that each non-zero $x \in \mathbb{H}$ has an inverse given by $x^{-1}=\frac{\bar{x}}{|x|^{2}}$.

In this paper we are going to consider polynomials $P$ whose coefficients are located on the left-hand side of the powers, i.e. $P$ has the form

$$
P(x)=a_{n} x^{n}+a_{n-1} x^{n-1}+\cdots+a_{1} x+a_{0}, \quad a_{k} \in \mathbb{H} .
$$

The set of such polynomials is a ring with respect to the operations of addition and multiplication defined in the same way as in the commutative case, where the variable $x$ is assumed to commute with the quaternion coefficients, i.e. if $Q(x)=b_{n} x^{n}+b_{n-1} x^{n-1}+\cdots+b_{1} x+b_{0}$, then

$$
P(x)+Q(x):=\sum_{k=0}^{\max \{m, n\}}\left(a_{k}+b_{k}\right) x^{k} \quad \text { and } \quad P(x) * Q(x):=\sum_{k=0}^{m+n}\left(\sum_{j=0}^{k} a_{j} b_{k-j}\right) x^{k} .
$$

We denote this ring by $\mathbb{H}[x]$ and call the polynomials one-sided left polynomials. In what follows we present some important definitions and well known properties of $\mathbb{H}[x]$ needed in the sequel.

Main definitions: Let $q$ be a quaternion and $P$ be a left one-sided polynomial.

1. Two quaternions $q$ and $q^{\prime}$ are called congruent, $q \sim q^{\prime}$, if there exists $h \neq 0$ such that $q^{\prime}=h q h^{-1}$.

2. The congruence, conjugacy or equivalence class of $q$, denoted by $[q]$, is the set $[q]=\left\{q^{\prime} \in \mathbb{H}: q \sim q^{\prime}\right\}$ which can be shown to be equal to $\left\{q^{\prime} \in \mathbb{H}: \operatorname{Re} q=\operatorname{Re} q^{\prime}\right.$ and $\left.|q|=\left|q^{\prime}\right|\right\}$.

3. The conjugate of $P$ is the polynomial $\bar{P}(x)=\bar{a}_{n} x^{n}+\bar{a}_{n-1} x^{n-1}+\cdots+\bar{a}_{1} x+\bar{a}_{0}$.

4. The characteristic polynomial of a quaternion $q$ is the polynomial

$$
\chi_{q}(x):=(x-q) *(x-\bar{q})=x^{2}-2 \operatorname{Re}(q) x+|q|^{2},
$$

which depends only on the real part and norm of $q$ and, if $q \in \mathbb{H} \backslash \mathbb{R}$, is an irreducible quadratic trinomial from the ring of polynomials $\mathbb{R}[x]$.

5. The evaluation of $P$ at $q$ is defined as $P(q):=a_{n} q^{n}+a_{n-1} q^{n-1}+\cdots+a_{1} q+a_{0}$.

6. A quaternion $q$ is a zero of $P$, if $P(q)=0$.

7. A zero $q$ is called an isolated zero of $P$, if $[q]$ contains no other zeros of $P$; if $q$ is not an isolated zero, then it is called a spherical zero of $P$ and $[q]$ is referred to as a sphere of zeros.

We list now the main results concerning the zero-structure and the factorization of polynomials in $\mathbb{H}[x]$. For the proofs and other details we refer to $[14,15]$. 
Theorem 1 (Euclidean division). If $A(x)$ and $B(x)$ are polynomials in $H[x]$ (with $\operatorname{deg} B \leq \operatorname{deg} A$ and $B \neq 0$ ), then there exist unique $Q(x)$ and $R(x)$ such that

$$
A(x)=Q(x) * B(x)+R(x)
$$

with $\operatorname{deg} R<\operatorname{deg} B$.

Theorem 2 (Fundamental Theorem of Algebra [16]). Any non-constant polynomial in $\mathbb{H}[x]$ always has a zero in $\mathbb{H}$.

Theorem 3 (Factorization into linear terms). Any monic polynomial $P$ of degree $n \in \mathbb{N}$ in $\mathbb{H}[x]$ admits a factorization into linear factors, i.e. there exist $x_{1}, \ldots, x_{n} \in \mathbb{H}$, such that

$$
P(x)=\left(x-x_{n}\right) *\left(x-x_{n-1}\right) * \cdots *\left(x-x_{1}\right) .
$$

Moreover, if $\mathbf{Z}_{P}$ denotes the zero-set of $P$, then

i. $\mathbf{Z}_{P} \subseteq \bigcup_{k=1}^{n}\left[x_{k}\right]$;

ii. each of the congruence classes $\left[x_{k}\right]$ contains (at least) a zero of $P$;

iii. if the congruence classes $\left[x_{k}\right]$ are distinct, then $P$ has exactly $n$ zeros $\zeta_{k}$ which are related to the factor-terms $x_{k}$ as follows:

$$
\begin{aligned}
\zeta_{1} & =x_{1}, \\
\zeta_{k} & =\bar{R}_{k}\left(x_{k}\right) x_{k}\left(\bar{R}_{k}\left(x_{k}\right)\right)^{-1} ; k=2, \ldots, n,
\end{aligned}
$$

where $R_{k}(x):=\left(x-x_{k-1}\right) * \cdots *\left(x-x_{1}\right)$.

We end the section by presenting several results which will play later on an important role on the identification of zeros and on the definition of multiple root.

Lemma 1 ([6]). Let $P \in \mathbb{H}[x], q \in \mathbb{H}$ and denote by $N(P)$ the $2 n$-th degree polynomial $P * \bar{P} \in \mathbb{R}[x]$. The following conditions are equivalent:

i. There is a $q^{\prime} \in[q]$ such that $P\left(q^{\prime}\right)=0$;

ii. The characteristic polynomial of $q, \chi_{q}$, is a divisor of $N(P)$;

iii. $q$ is a root of $N(P)$.

Lemma 2 ([6, 17]). A non-real zero $q$ is a spherical zero of $P \in \mathbb{H}[x]$ if and only if any of the following equivalent conditions hold:

i. $q$ and $\bar{q}$ are both zeros of $P$;

ii. $[q] \subseteq \mathbf{Z}_{P}$;

iii. $\chi_{q}$ is a divisor of $P$.

Lemma 3 ([18]). Consider a quadratic polynomial factorized in the form $P(x)=\left(x-x_{2}\right) *\left(x-x_{1}\right)$, where $x_{1}, x_{2} \in \mathbb{H} \backslash \mathbb{R}$ and $\left[x_{1}\right]=\left[x_{2}\right]$.

i. If $x_{1} \neq \bar{x}_{2}$, then the only zero of $P$ is $x_{1}$.

ii. If $x_{1}=\bar{x}_{2}$, then $x_{1}$ is a spherical zero.

Lemma $4([6,7])$. Let $P \in \mathbb{H}[x]$ have degree $n$. Then $x_{1} \in \mathbb{H} \backslash \mathbb{R}$ is the unique zero of $P$ if and only if $P$ admits a unique factorization which has the form

$$
P(x)=\left(x-x_{n}\right) * \cdots *\left(x-x_{2}\right) *\left(x-x_{1}\right), x_{l} \in\left[x_{1}\right], x_{l} \neq \bar{x}_{l-1}, ; l=2, \ldots, n .
$$




\section{Multiplicity in $\mathbb{H}[x]$}

In this section we present and analyze different, not always equivalent, definitions of multiplicity of a root of a quaternionic polynomial that have been introduced over the years. In what follows, $P$ denotes a quaternionic left one-sided polynomial of degree $n$.

The first definition we present seems, at the first sight, to be the most natural, as it is a simple adaptation of the complex case; this is the notion of multiplicity considered in $[3,4,5]$.

Definition $1([3,4,5]) \quad$ The multiplicity $m_{P}(q)$ of a root $q$ of $P$ is defined in the following equivalent ways:

1. The largest $k \in \mathbb{N}$ for which $(x-q)^{* k}$ divides $P$, i.e. $P$ can be written as

$$
P(x)=Q(x) *(x-q)^{* k}
$$

for some polynomial $Q$. We adopt the notation $(x-q)^{* k}$ (cf. [18]) to represent the product of the $k$ factors $(x-q) * \cdots *(x-q)$.

2. The least positive integer $k$ such that the $k$-th formal derivative of $P$ does not vanish at $q$.

In the book [18], $m_{P}(q)$ is called the classical multiplicity of $q$. Observe that if $P=\chi_{q}^{n}$, then $m_{P}(q)=n$.

Since the non-real zeros of a quaternionic polynomial can be non-isolated, it is natural to have a notion of multiplicity for conjugacy classes.

Definition $2([\mathbf{8}, \mathbf{4}, \mathbf{5}]) \quad$ Let $[q]$ be the conjugacy class of a non-real quaternion $q$; the multiplicity $\mathcal{M}_{P}([q])$ of $[q]$ as a sphere of zeros of $P$ is given by the following equivalent definitions:

1. The largest $k \in \mathbb{N}_{0}$ for which $\chi_{q}^{k}$ divides $P$.

2. The largest $k \in \mathbb{N}_{0}$ for which $\chi_{q}^{2 k}$ divides $N(P)$.

3. $\min _{q^{\prime} \in[q]}\left\{m_{P}\left(q^{\prime}\right)\right\}$.

Although, at a first reading, the following two (different, but equivalent) definitions of multiplicity in Definition 3 might not seem very natural, it turns out that they are, in fact, more appropriate, in the quaternionic context, than the (classical) definition of multiplicity $m_{p}(q)$. It is interesting to observe that these definitions of multiplicity predate the notion of multiplicity given in Definition 1 . The first definition was given by Pereira [7] and is presented here as it is stated in the original, while the second one, due to Beck [6], is rephrased in the spirit of the present paper.

Definition $3([6,7]) \quad$ The multiplicity $\mu_{P}(q)$ of a root $q$ of $P$ is defined in the following equivalent ways:

1. The maximum degree of the right factors of $P$ having $q$ as their unique zero.

2. Let $k \in \mathbb{N}_{0}$ be the largest value for which $P(x)$ can be written as

$$
P(x)=Q(x) * \chi_{q}^{k},
$$

for some polynomial $Q$ and let

$$
M_{Q}(q):=\max \left\{k \in \mathbb{N}_{0}: \chi_{q}^{k} \text { divides } N(Q)\right\} .
$$

Then

$$
\mu_{P}(q):=k+M_{Q}(q)
$$


Table 2: On the theoretical notion of multiplicity

\begin{tabular}{|c|c|c|c|c|c|c|}
\hline & $p_{1}(x)$ & $p_{2}(x)$ & $p_{3}(x)$ & $p_{4}(x)$ & $p_{5}(x)$ & $p_{6}(x)$ \\
\hline$m_{P}(\mathbf{i})$ & 2 & 1 & 1 & 2 & 2 & 2 \\
$\mathcal{M}_{P}([\mathbf{i}])$ & 0 & 0 & 1 & 1 & 0 & 2 \\
$\mu_{P}(\mathbf{i})$ & 2 & 2 & 1 & 2 & 3 & 2 \\
$\mathcal{I}_{P}(\mathbf{i})$ & 2 & 2 & 0 & 1 & 3 & 0 \\
$\nu_{P}(\mathbf{i})$ & 2 & 2 & 2 & 3 & 3 & 4 \\
\hline
\end{tabular}

We underline that if $q$ is an isolated root of $P$, then in (7) we have $k=0$ and $P=Q$ and hence, we obtain $\mu_{P}(q)=M_{P}(q)$. In fact (8) appears in the work [8] as the definition of multiplicity for isolated roots. Observe also that if $q$ is a root of the factor polynomial $Q$ in (7), then it is necessarily an isolated root of $Q$. In [18], the authors called $M_{Q}(q)$ the isolated multiplicity of $q$ as a root of $P$ which we are going to denote here by $\mathcal{I}_{P}(q)$. In this paper, a spherical root of $P$ which is also an isolated root of $Q$ will be called a mixed root of $P$.

We finalize with another notion of multiplicity that was considered in $[19,20]$.

Definition $4([19,20]) \quad$ Let $q$ be a zero of a polynomial $P$. The multiplicity $\nu_{P}(q)$ of $q$ is given by the following equivalent definitions:

1. The number of factors of the form $\left(x-q^{\prime}\right)$, with $q^{\prime} \in[q]$, in any factorization of $P$ into monic irreducible factors.

2. The largest $k \in \mathbb{N}$ for which $\chi_{q}^{k}$ divides $N(P)$.

Obviously $\nu_{P}(q)=\mu_{P}(q)$, for isolated roots, whereas in the case of spherical (non-mixed) roots one has $\nu_{P}(q)=2 \mu_{P}(q)=2 \mathcal{M}_{P}([q])$.

Example 1. 1 We illustrate the previously introduced definitions of multiplicity by considering the polynomials:

$$
\begin{array}{lll}
p_{1}(x)=(x-\mathbf{i})^{* 2}, & p_{2}(x)=(x-\mathbf{j}) *(x-\mathbf{i}), & p_{3}(x)=(x+\mathbf{i}) *(x-\mathbf{i}), \\
p_{4}(x)=(x+\mathbf{i}) *(x-\mathbf{i})^{* 2}, & p_{5}(x)=(x-\mathbf{j}) *(x-\mathbf{i}) * 2, & p_{6}(x)=(x+\mathbf{i})^{* 2} *(x-\mathbf{i}) * 2
\end{array} .
$$

We point out that $\mathbf{i}$ is an isolated zero of $p_{1}, p_{2}$ and $p_{5}$, while [i] is a sphere of zeros of $p_{3}, p_{4}$ and $p_{6}$. Polynomial $p_{4}$ also has $\mathbf{i}$ as a mixed zero.

The results presented in Table 2 show that there are no repeated rows which reveals that the definitions are all essentially different.

\section{The numerical point of view}

In this section we analyze the behavior of Newton's method when applied to polynomials with multiple roots and use the basin of attraction of those roots as one of the tools for characterizing such roots.

We recall that the quaternionic equation $P(x)=P\left(x_{0}+x_{1} \mathbf{i}+x_{2} \mathbf{j}+x_{3} \mathbf{k}\right)=0, P \in \mathbb{H}[x]$ can be written as a system of four nonlinear equations

$$
\boldsymbol{P}(\boldsymbol{x})=\left(p_{0}\left(x_{0}, x_{1}, x_{2}, x_{3}\right), p_{1}\left(x_{0}, x_{1}, x_{2}, x_{3}\right), p_{2}\left(x_{0}, x_{1}, x_{2}, x_{3}\right), p_{3}\left(x_{0}, x_{1}, x_{2}, x_{3}\right)\right)=\mathbf{0},
$$

for which Newton's iterative scheme (cf. (2)) reads as

$$
4 \mathrm{D}-\mathrm{NM}: \quad \boldsymbol{z}_{k+1}=\boldsymbol{z}_{k}-\left(J \boldsymbol{P}\left(\boldsymbol{z}_{k}\right)\right)^{-1} \boldsymbol{P}\left(\boldsymbol{z}_{k}\right), \quad \boldsymbol{z}_{0}=\boldsymbol{c} .
$$


Remark 1. We could have used quaternionic versions of Newton's method in our analysis, but since this requires extra assumptions on the initial guesses (see [13] for details), we decided to restrict our discussion to the iterative scheme (9). Our intention here is to focus on issues related to different notions of multiplicity and to not bring to light convergence issues of the quaternionic Newton methods.

We illustrate the behavior of the method by considering polynomials whose zero set is $\{0, \mathbf{i}\}$ or $\{0\} \cup[\mathbf{i}]$ and choosing the initial guesses on three different domains, all containing the segment $[0, \mathbf{i}]$ :

$\Omega_{1}=\{(x, y, 0,0): x, y \in[-2,2]\}, \quad \Omega_{2}=\{(x, y, x, 0): x, y \in[-2,2]\}, \quad \Omega_{3}=\{(0, x, y, 0): x, y \in[-2,2]\}$.

We use as stopping criteria the incremental sizes and the number of iterations, i.e. the iterative process stops whenever two consecutive estimates differ by less than $10^{-3}$ or the number of iterations reaches 30 (for our purpose here it is not worth to ask for more accuracy).

We also make use of the so-called computational order of convergence $\rho$ of Newton's method to identify the cases where its "expected" quadratic order of convergence is lost, revealing in this way the characteristic behavior of the method in the presence of multiple roots. At the end of the section we present estimates for $\rho$ based on the use of the approximations (see e.g. [21])

$$
\rho \approx \frac{\log \left(\left|z^{*}-z_{k+1}\right| /\left|z^{*}-z_{k}\right|\right)}{\log \left(\left|z^{*}-z_{k}\right| /\left|z^{*}-z_{k-1}\right|\right)} \quad \text { or } \quad \rho \approx \frac{\log \left(|| z^{*}|-| z_{k+1}|| /|| z^{*}|-| z_{k}||\right)}{\log \left(|| z^{*}|-| z_{k}|| /|| z^{*}|-| z_{k-1}||\right)}
$$

where $z_{k-1}, z_{k}, z_{k+1}$ are three consecutive iterations close to the isolated root $z^{*}$ or to the sphere of zeros $\left[z^{*}\right]$, respectively. We have generated lists of 100 random initial guesses close to a zero and/or sphere of zeros of each of the polynomials under consideration and present the mean $\tilde{\rho}\left(z^{*}\right)$ and/or $\tilde{\rho}\left(\left[z^{*}\right]\right)$ of the observed values.

Example 2. 2 Consider the polynomials

$$
s_{1}(x)=x *(x-\mathbf{i}), \quad s_{2}(x)=x *(x-\mathbf{i})^{* 2}, \quad s_{3}(x)=x *(x-\mathbf{i})^{* 3}
$$

on its expanded form. Observe that these polynomials have 0 and $\mathbf{i}$ as (isolated) roots and that in $\Omega_{1}$ these polynomials coincide with the complex polynomials used in Table 1. Moreover, $m_{s_{i}}(\mathbf{i})=\mu_{s_{i}}(\mathbf{i})=\mathcal{I}_{s_{i}}(\mathbf{i})=$ $\nu_{s_{i}}(\mathbf{i})=i(i=1,2,3)$.

Table 3 contains the basins of attraction of 0 (in red) and $\mathbf{i}$ (in blue). We can observe that the number of iterations increases with $i$, which means that the quadratic convergence of Newton's method seems to be lost $(i=2,3)$. This can be confirmed from the computational order of convergence presented in Table 6. Of course that this is not enough to conclude that $\mathbf{i}$ is a double (resp. triple) root of $s_{2}$ (resp. $s_{3}$ ), as we know it is true in $\mathbb{C}$ (see also Remark 2). On the other hand, the idea that the basin of attraction of the multiple root "invades" the other basin is not visible in $\Omega_{2}$ and $\Omega_{3}$.

\section{Example 3. 3 The polynomials}

$$
s_{4}(x)=x *(x+\mathbf{i}) *(x-\mathbf{i}), \quad s_{5}(x)=x *(x+\mathbf{i}) *(x-\mathbf{i})^{* 2}, \quad s_{6}(x)=x *(x+\mathbf{i})^{* 2} *(x-\mathbf{i})^{* 2} .
$$

all have the sphere of zeros $[\mathbf{i}]$, but the zero $\mathbf{i}$ is a mixed zero of $s_{5}$, since $s_{5}(x)=x *(x-\mathbf{i}) * \chi_{\mathbf{i}}$. The theoretical multiplicities of $\mathbf{i}$ have been considered already in Example 1 (see Table 2, polynomials $p_{3}, p_{4}$ and $\left.p_{6}\right)$. The numerical results presented in Table 4 show also the set of initial points converging to a root in [i ] different from $\mathbf{i}$. We call such set the basin of attraction of $[\mathbf{i}] \backslash\{\mathbf{i}\}$. We kept the color scheme of Example 2 and added the orange color for the basin of attraction of $[\mathbf{i}] \backslash\{\mathbf{i}\}$. We can see that for the polynomial $s_{4}$ all the roots seem to be simple, but the basin of attraction of $\mathbf{i}$ as a root of $s_{5}$ (lighter blue) reveals a nature different from the roots in $[\mathbf{i}] \backslash\{\mathbf{i}\}$ (dark orange). For polynomial $p_{6}$ we can also observe lighter orange basins, suggesting the double multiplicity of the spherical root (cf. Table 6 and 7).

Example 4. 4 Our last example concerns the following polynomials

$s_{7}(x)=x *(x-\mathbf{j}) *(x-\mathbf{i}), \quad s_{8}(x)=x *(x-\mathbf{j}) *(x-\mathbf{i})^{* 2}, \quad s_{9}(x)=x *(x-\mathbf{k}) *(x-\mathbf{j}) *(x-\mathbf{i})$ 
Table 3: Newton's method applied to polynomials with $m_{s_{i}}(\mathbf{i})=i$

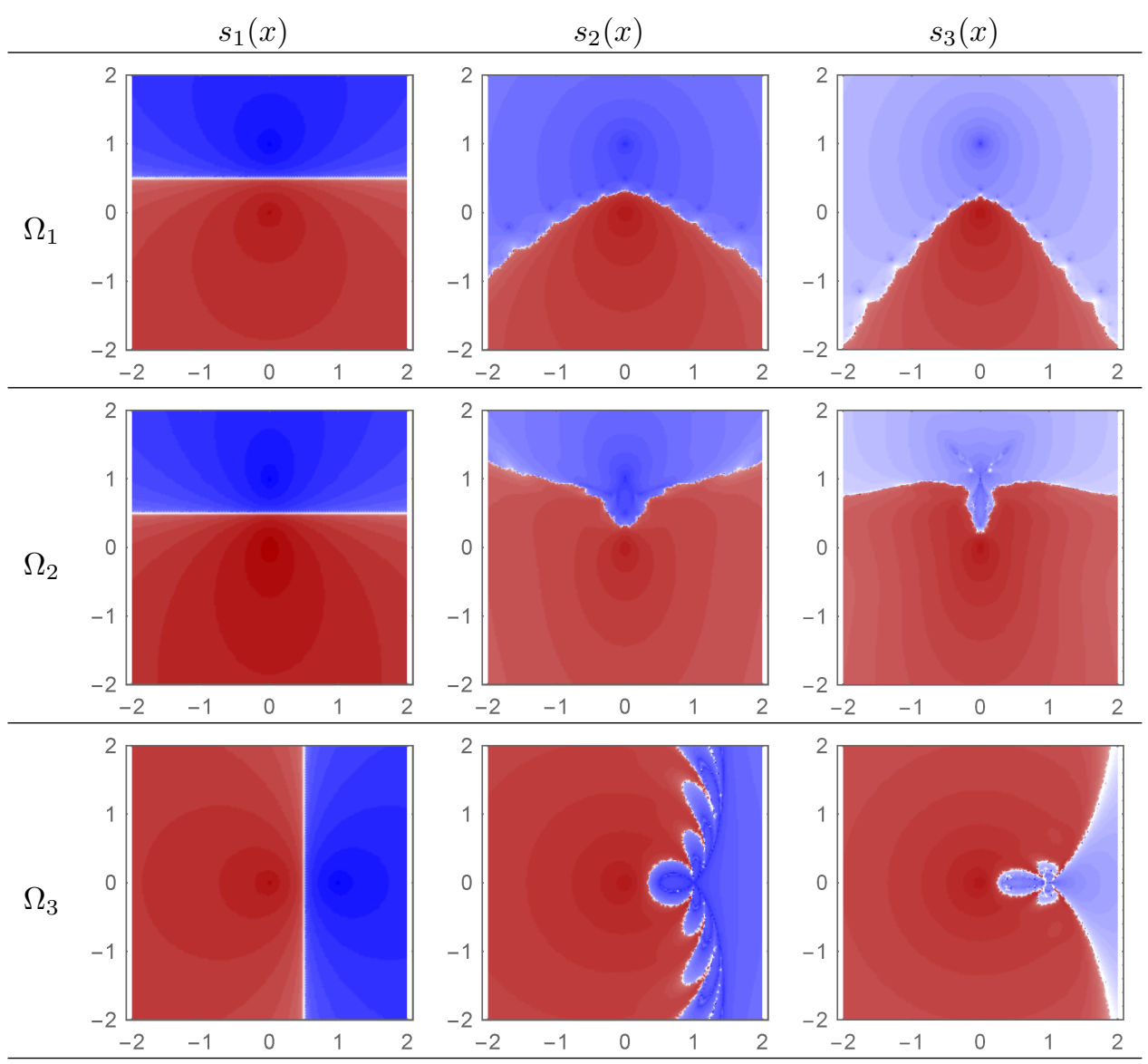


Table 4: Newton's method applied to polynomials with a spherical root

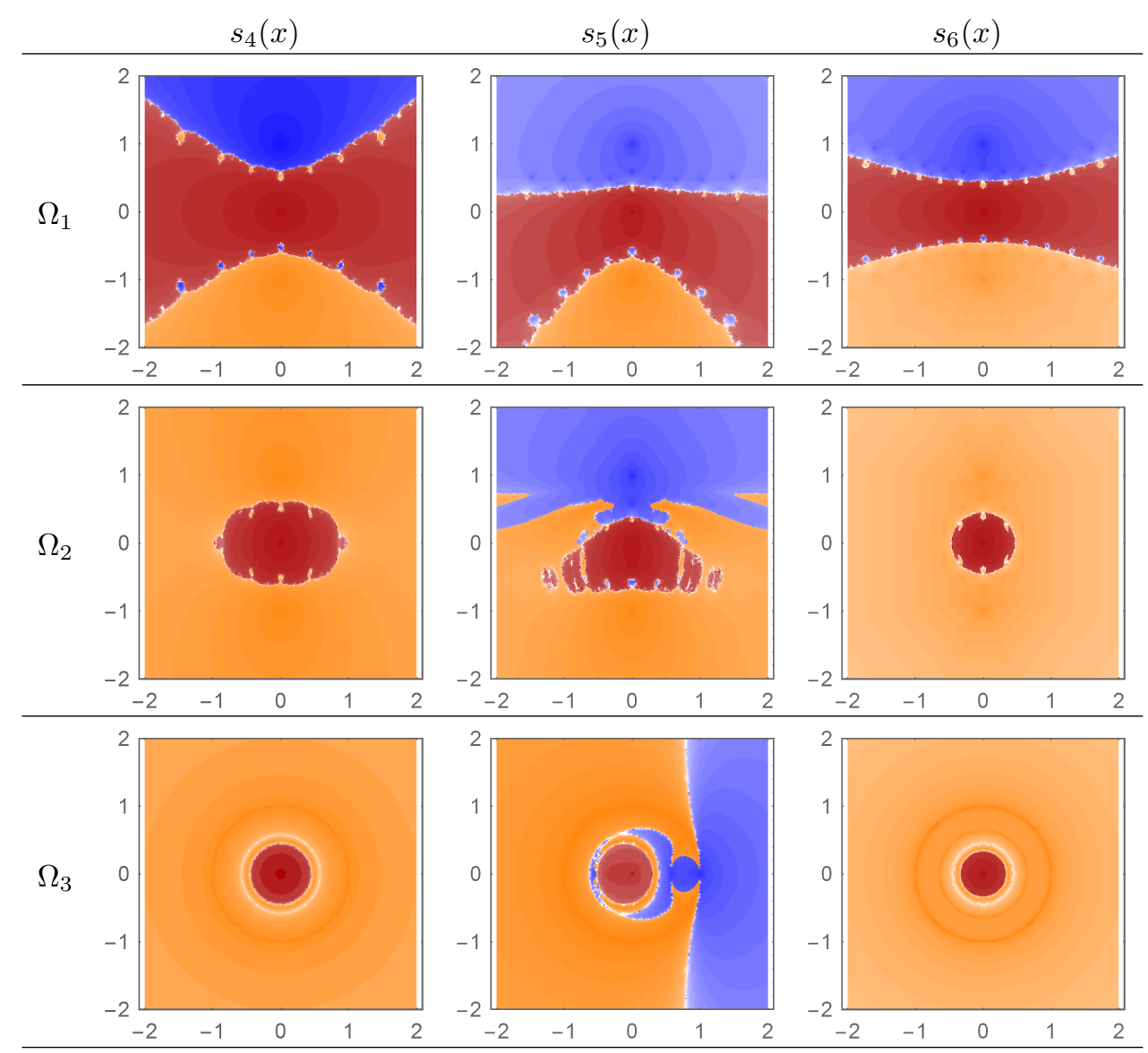


Table 5: Newton's method applied to polynomials with roots with non classical multiplicity
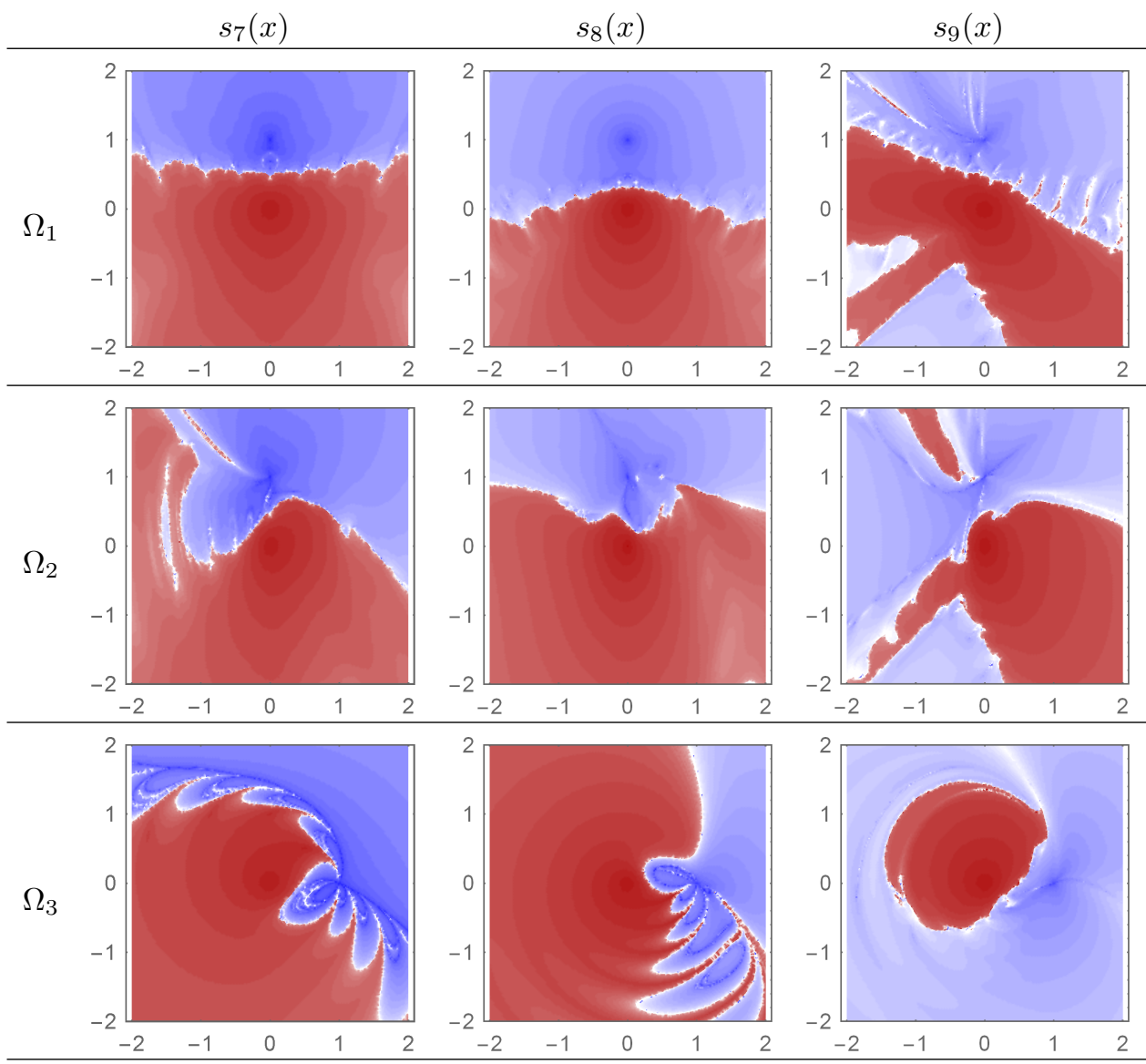

which share the property that $m_{s_{k}}(\mathbf{i}) \neq \mu_{s_{k}}(\mathbf{i})$ and $\mathcal{M}_{s_{k}}([\mathbf{i}])=0,(k=7,8,9)$. As can be seen in Table 5, the patterns are very different from the complex case, which make it difficult to reason about the link between the multiplicity of the roots and the corresponding basins of attraction of Newton's method. However one can see that the color intensity suggests that $\mathbf{i}$ is not a simple root. Table 6 lends support to this idea (see also Table 7).

Remark 2. One of the traditional ways of re-establishing the quadratic order of convergence of Newton's method to a root of multiplicity $m$, is to use the modified scheme

$$
\mathrm{MNM}: \quad \boldsymbol{z}_{k+1}=\boldsymbol{z}_{k}-m\left(J \boldsymbol{p}\left(\boldsymbol{z}_{k}\right)\right)^{-1} \boldsymbol{p}\left(\boldsymbol{z}_{k}\right), \quad \boldsymbol{z}_{0}=\boldsymbol{c},
$$

usually known as relaxed Newton's method. This modification speeds up the convergence to a root with multiplicity exactly $m$, but the convergence to roots with different multiplicity is affected (see e.g. [22]). Table 7 contains the results of applying this scheme to the polynomials $s_{2}, s_{3}$ and $s_{6}$, with $m=2,3$ and 2, respectively. This modification does not work for the other cases, revealing that the problem of finding appropriated modifications to Newton's method in order to recover its order of convergence is much more demanding in $\mathbb{H}[x]$. Further investigations on this subject have to be done.

\section{Conclusions and final remarks}

In this paper we have collected different concepts of multiplicity of a root of a quaternionic one-sided left polynomial that we have found in the literature. We performed several experiments to understand the behavior 
Table 6: Computational order of convergence of Newton's method

\begin{tabular}{cccccccccc} 
& $s_{1}(x)$ & $s_{2}(x)$ & $s_{3}(x)$ & $s_{4}(x)$ & $s_{5}(x)$ & $s_{6}(x)$ & $s_{7}(x)$ & $s_{8}(x)$ & $s_{9}(x)$ \\
\hline$\tilde{\rho}(0)$ & 2.00 & 2.00 & 2.00 & 3.00 & 2.00 & 3.00 & 2.00 & 2.00 & 2.00 \\
$\tilde{\rho}(\mathbf{i})$ & 2.00 & 1.00 & 0.94 & -- & 1.00 & -- & 1.00 & 1.00 & 1.00 \\
$\tilde{\rho}([\mathbf{i}])$ & -- & -- & -- & 2.02 & 2.06 & 0.99 & -- & -- & -- \\
\hline
\end{tabular}

Table 7: Relaxed Newton's method

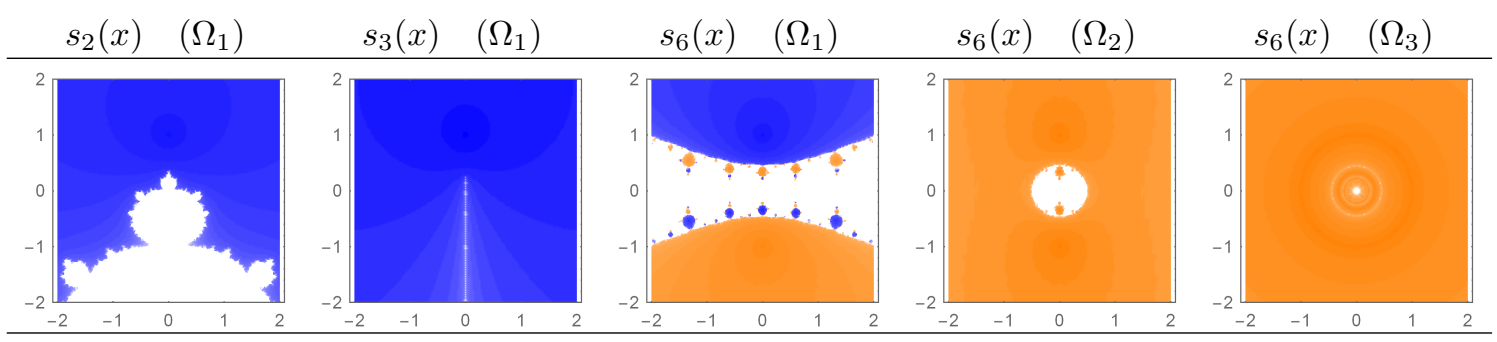

of the well-known Newton's method for systems of nonlinear equations. Two key-aspects were considered in our discussion: the basin of attraction of the roots and the computational order of convergence of the iterative scheme. Taking into account all these facts, we can say that the definition $\mu_{P}(q)$ given in $[6,7]$ is the one that best reflects the computational point of view of Newton's method.

The study of the basins of attraction of a root can reveal important aspects of a root-finding method. In the present context, it seems to us that a deeper analysis has to be done, not only by its own interest, but also because it can shed light on modifications to re-establish the quadratic order of convergence of Newton's method.

\section{Acknowledgments}

Research at CMAT was financed by Portuguese Funds through FCT - Fundação para a Ciência e a Tecnologia, within the Project UID/MAT/00013/2013. Research at NIPE has been carried out within the funding with COMPETE reference number POCI-01-0145-FEDER-006683, with the FCT/MEC's (Fundação para a Ciência e a Tecnologia, I.P.) financial support through national funding and by the ERDF through the Operational Programme on "Competitiveness and Internationalization - COMPETE 2020" under the PT2020 Partnership Agreement.

\section{References}

[1] J. M. McNamee, Numerical methods for roots of polynomials, Part I, Vol. 16 (Elsevier B. V., Amsterdam, 2007).

[2] F. Miranda and M. I. Falcão, Lecture Notes in Comput. Sci. 8579, 146-161 (2014).

[3] G. Gentili and C. Stoppato, Michigan Mathematical Journal 56, 655-667 (2008).

[4] B. Kalantari, J. Complexity 29, 302-322 (2013).

[5] V. Bolotnikov, arXiv:1505.03573 (2015).

[6] B. Beck, Enseign. Math. (2) 25, 193-201 (1979). 
[7] R. Pereira, "Quaternionic polynomials and behavioral systems," Ph.D. thesis, Universidade de Aveiro 2006.

[8] N. Topuridze, Journal of Mathematical Sciences 160, 843-855 (2009).

[9] G. Gentili and D. C. Struppa, Milan J. Math. 76, 15-25 (2008).

[10] M. Scott, B. Neta, and C. Chun, Applied Mathematics and Computation 218, 2584 - 2599 (2011).

[11] J. M. Gutiérrez, L. J. Hernández-Paricio, M. Marañón-Grandes, and M. T. Rivas-Rodríguez, Numerical Algorithms 66, 431-455 (2014).

[12] B. D. Stewart, Attractor basins of various root-finding methods, Master's thesis, Naval Postgraduate School, Monterey, California (2001).

[13] M. I. Falcão, Appl. Math. Comput. 236, 458-470 (2014).

[14] B. Gordon and T. Motzkin, Trans. Amer. Math. Soc. 116, 218-226 (1965).

[15] T.-Y. Lam, A first course in noncommutative rings, Graduate Texts in Mathematics (Springer-Verlag, New York, 1991).

[16] I. Niven, Amer. Math. Monthly 48, 654-661 (1941).

[17] A. Pogorui and M. Shapiro, Complex Var. Theory Appl. 49, 379-389 (2004).

[18] G. Gentili, C. Stoppato, and D. C. Struppa, Regular Functions of a Quaternionic Variable, Springer Monographs in Mathematics (Springer-Verlag Berlin Heidelberg, 2013).

[19] U. Bray and G. Whaples, Canad. J. Math. 35, 509-515 (1983).

[20] R. Ghiloni and A. Perotti, Ann. Mat. Pura Appl. (4) 190, 539-551 (2011).

[21] A. Y. Ozban, Appl. Math. Lett. 17, 677-682 (2004).

[22] W. Gilbert, Fractals 9, 251-262 (2001). 